\title{
$\mathfrak{I} \mathfrak{n} \mathfrak{h} \mathfrak{a l t}$.
}

\section{B̈̈rgerlide Redt.}

\section{Ineidyadyt.}

1. Unfäle wäbreno der Bwangäberwaltung. Wer haftet für bie Bejeitigung

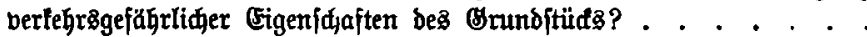

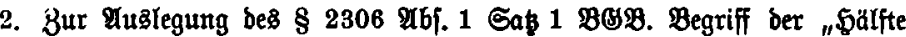

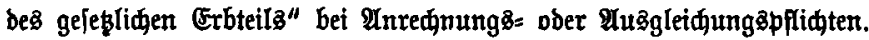

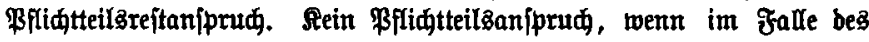

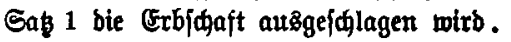

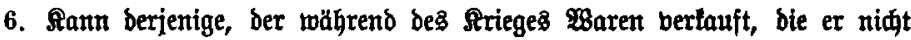
befißt, fid auf unmögliøłfeit berufen, wenn feine Gintermänner infolge

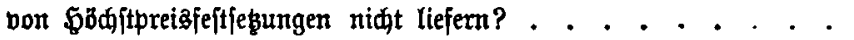

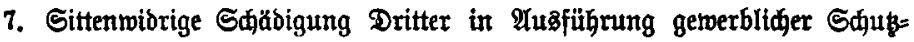
verträge . . . . . . . . . . . . . . . . . . .

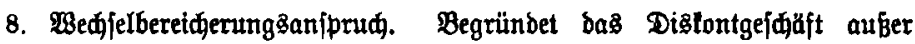

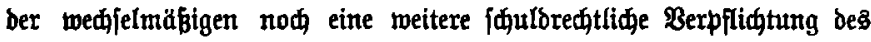
Beđ̆flelgeberō? . . . . . . . . . . . . . . . . . .

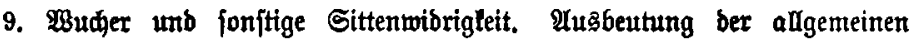

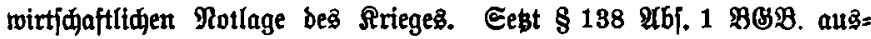
nahmolos ein \$andeln beiber Teile gegen bie guten Sitten vorauz? .

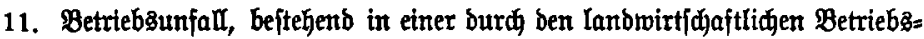

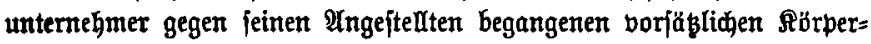
verlefung. . . . . . . . . . . . . . . . . . . . 33

13. 3ur frage ber Shabensbegrïndung aus ber Berfon eines Dritten .

14. $\mathfrak{M u k}$ ber gutgläubige $\mathfrak{g}$ hpothetenerwerber, deffen Bormann burd den

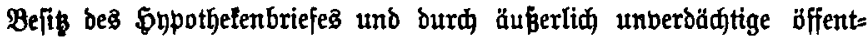

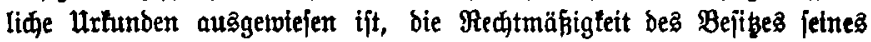
Bormanns und bie Eattheit ber Urfunden nauprüfen? . . . . .

15. Wann ift ber Răufer, bem eine andere als bie bedungente $\mathfrak{B a r e}$ geliefert wirb, bon ber aus $\$ 378 \mathfrak{\$ B Q B}$. folgenben Unterjudung pflint befreit?

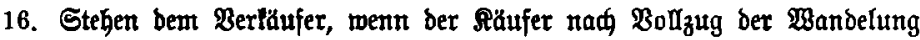

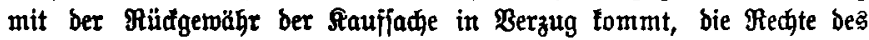
$\S 326 \mathfrak{B G S}$. zu? . 
ixt.

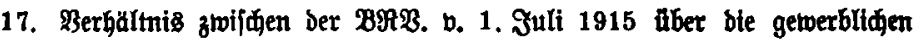

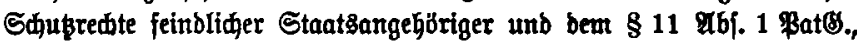
Faffung v. 6. Juni 1911 . . . . . . . . . . . . . .

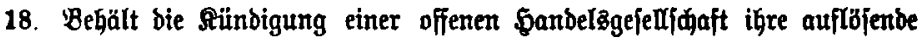

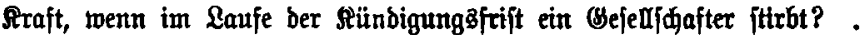

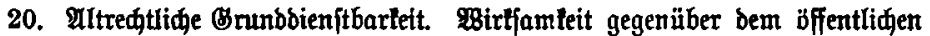
BIauben bes Bronbbử

21. Bum Begriff ber höheren Bewalt i. S. bes \$aftpflidtgejęzes . . .

22. Berleģt ein mit ber \$erftellung einer öffentliden ober öffentlid be glaubigten Urhunde ż山 einer Regiftereintragung beauftragter Rotar feine Amtapflid)t ober eine Bertragß̈pflidt, wenn ex bie Urtunbe unridtig abfaß̧t ober veripätet bei Bseridt einreidt? . . . . . . . . .

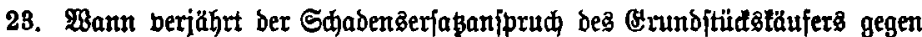

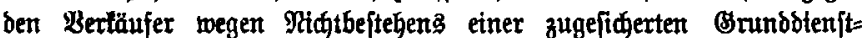
bartett? . . . . . . . . . . . . . . . . . . .

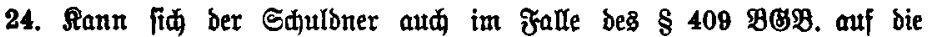

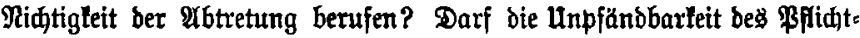

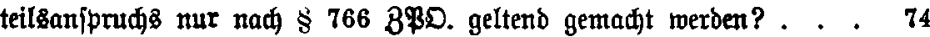

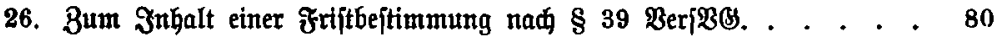

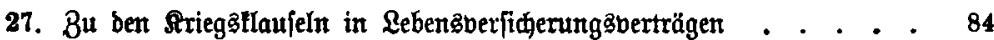

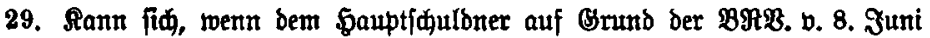
1916 eine Bahlutggfrift betwilligt ift, au出 ber Bürge barauf berufen?

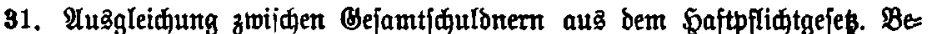
rüuffidtigung ber Erhähung Der Betriebsిgefahr burd Säumnia eineå

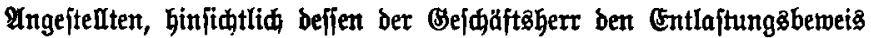
gefübrt bat . . . . . . . . . . . . . . . . . 96

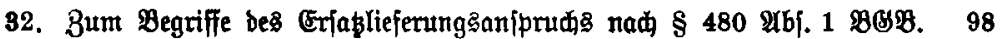

83. Stauanlagen für $\mathfrak{W a f f e r t r i e b w e r t e . ~ " B e j o n d e r e r " ~ p r i b a t r e d t l i z i d e r ~ T i t e l ~}$

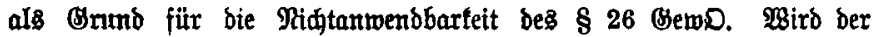

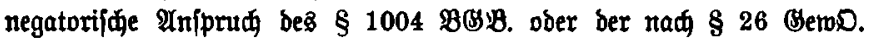

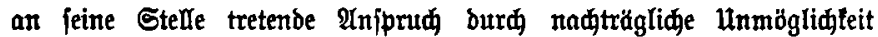

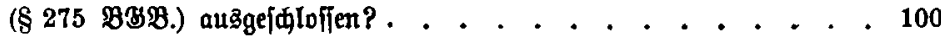

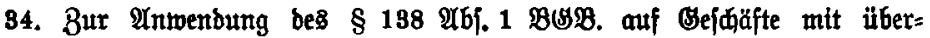
mäßjigem Geminn (丹rieggimuder) . . . . . . . . . . . . .

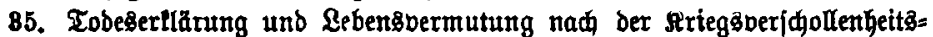

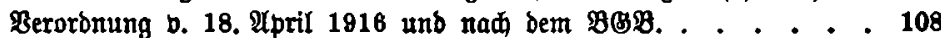

86. Bezugnahme bes Aonnoffements auf bie Ehartepartie. Cinfügung bon

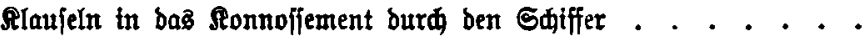

B7. Wirłungen einer zugunjten eine nađjtełenden Sypothetengläutbigets eingetragenen \&bjhungsoormerlung, bie ben Fall ber Nidtentitehung ber §ypotbelenforberung mitumfaßt. Sft biejer Fall gegeben, wenn 
st.

bex Brunbitüdß̊eigentî̉mer ben Cintritt ber Bebingung, unter ber eine Forberung burd bie borbergehenbe \$ypotget gefidert werben follte, wiber Treu unb Blauben verbinbert hat?

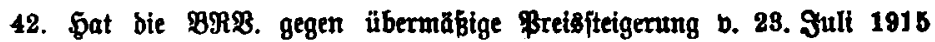

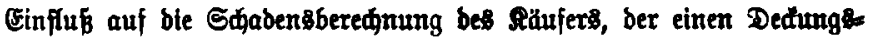
lauf borgenommen hat?. . . . . . . . . . . . . . .

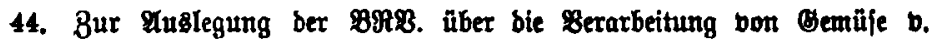

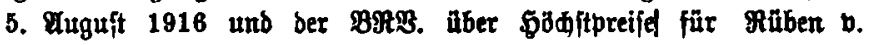
26. Dttober 1916 . . . . . . . . . . . . . . . . . . 138

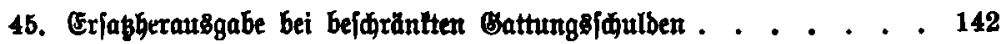

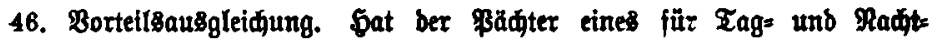

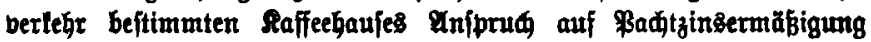

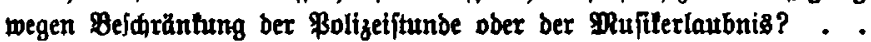

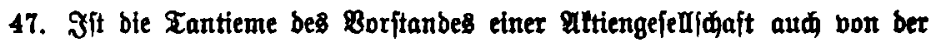
Sonberrüdlage zur Slळerung ber Ariegsgeminnfteuer zu entriळten? .

48. Jit im falle einer unter bie Beitberfiđerung gegen Srieggyefahr fallenben Qujbringung bie \$rämic bis zur Ronbemnation fortzuzablen? . . 150

51. Bur Inwentung bęి $\$ 477$ घbГ. 3 B\&B. . . . . . . . . . . 158

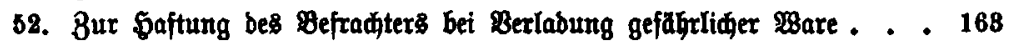

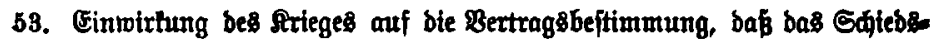
getidat und bas Medt eines feinbliden Staates majgebend fein follen. Bum Befahrübergang bei überjeeijळen Staufgeiळäften. Bedeutung be Begriff "babarie" . . . . . . . . . . . . . . . 166

54. Inwenbungsgebiet bex Raufpertragstlaufel "glïdlide Antunft vos behalten". . . . . . . . . . . . . . . . . . . . .

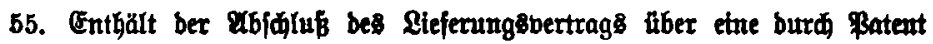

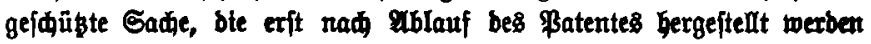
joIl, eine \$atentberlęung?. . . . . . . . . . . . . . . 172

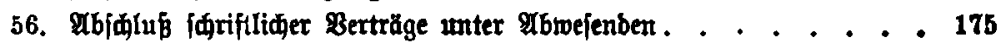

57. Jă welden Boridriften Gaftet bie Gijenbabn fïr ben Berluft ber bis

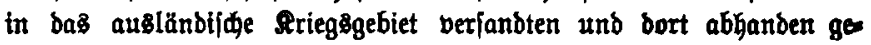

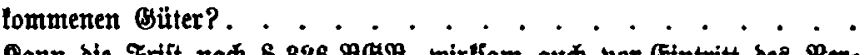

58. Tann bie Frift nad $\$ 326$ B(8B. wirlfam aud vor (Eintritt bes Bet= zug des Sful'ners beftimmt werben? . . . . . . . . . . 180

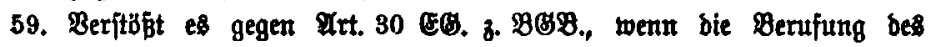

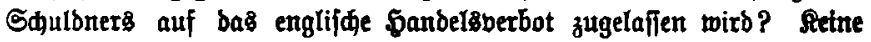

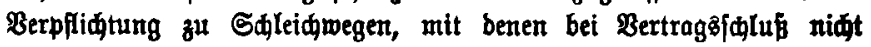
gereqnet wurbe. . . . . . . . . . . . . . . . . .

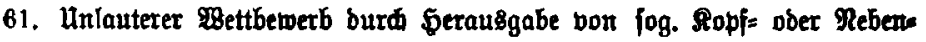
blättern einer 8eitung, bie bur屯 Benennung na出 einem beftimmten Orte ben Gindrud eines Lolalblattes exweden 
Jntralt.

Rx.

Gelte

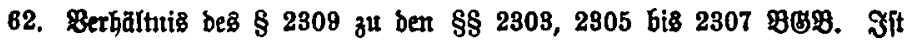

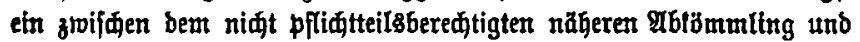

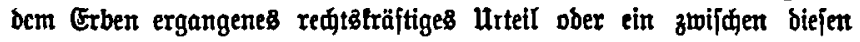

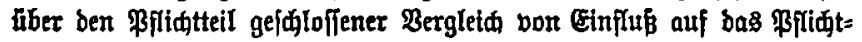

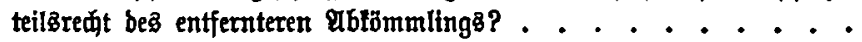

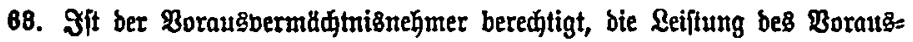

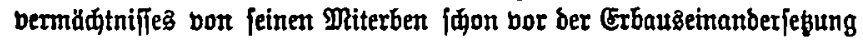
zu forbern?. . . . . . . . . . . . . . . . . . . .

64. In

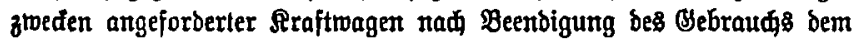

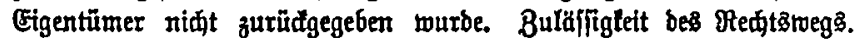
\$aftung bes Beamten gegenüber bem Eigentümer bes \$agens . . .

66. Berftoß gegen bie guten Sitten burd Bereinbarung eines unberbăltni\&= măßsig hołen Mällerlohns für bie Bermittelung von Rriegslieferungen

69. Eritrečt fid ber Frormzwang bes § 313 BBB. aud auf bie zum Ent= gelt für bie Figentumüübertragung übernommene Berpflidtung, eine

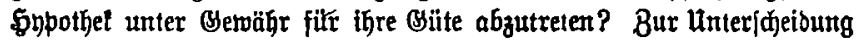

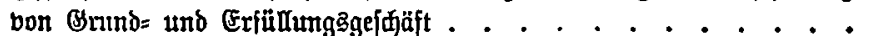

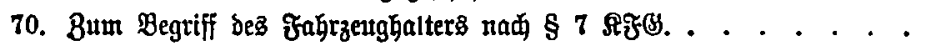

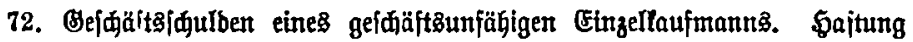

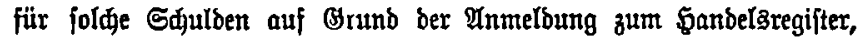

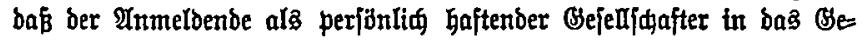
fäät eingetreten fei . . . . . . . . . . . . . . . .

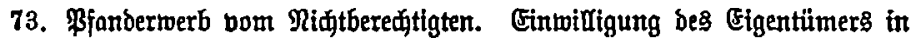
Sie Berpfändung von \$ertpapieren burd einen Drittent . . . . .

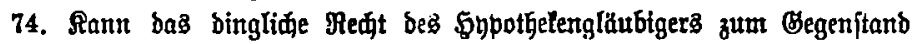
einer \$ärgidaft gemadt werden? Bur frrage ber reformatio in peius

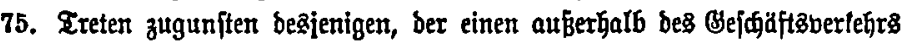

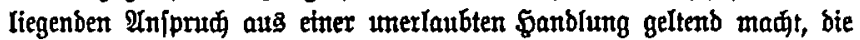

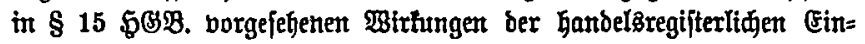
tragung eir? . . . . . . . . . . . . . . . . . .

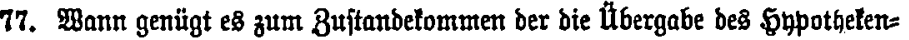
briefs exfesentent Bereinbarung, daß̧ bie bem Beffionar vom Bedenten

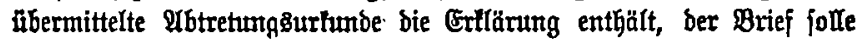
bem Bejīionar ausigebänbigt werben? Rann ber Bebent feine Ertlärung nặträglid bem Brumbbudante gegenüber miberrufen? . . . .

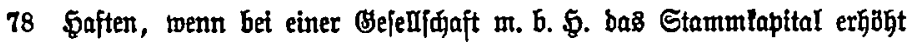

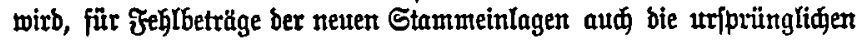

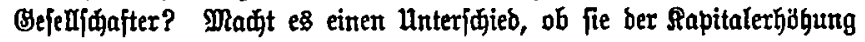
zugeftimmt baben ober nifut? . . . . . . . . . . . 251

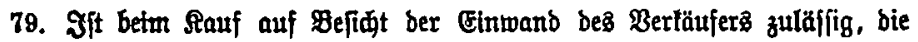




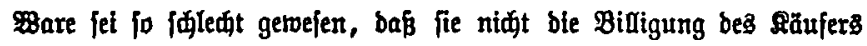
gefunden haben mürde? ..................

83. Finben bie Beftimmungen über ben Єăulbnernerzug auf einen $\mathfrak{B e}=$

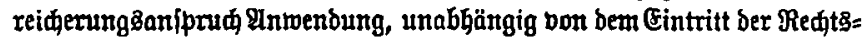

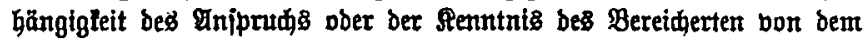

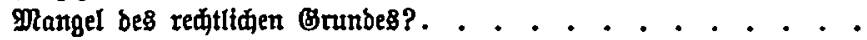
254

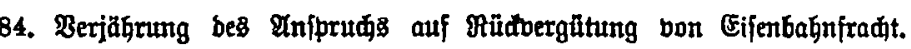

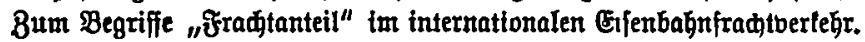

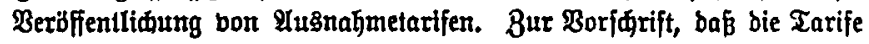

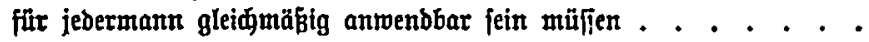

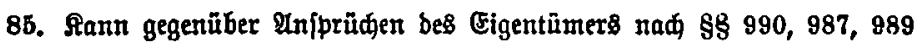

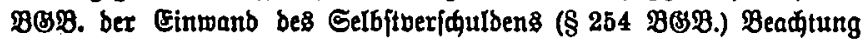

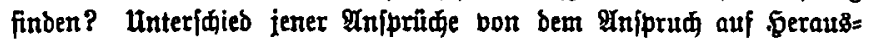
gabe (\$ 985 :SSP.) . . . . . . . . . . . . . . . . .

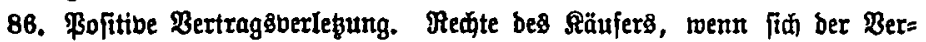

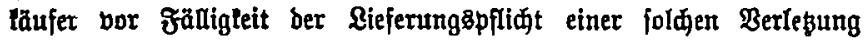
佸ulbig madt . . . . . . . . . . . . . . . . . . .

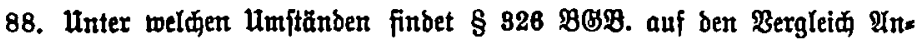
wenbung? . . . . . . . . . . . . . . . . . . .

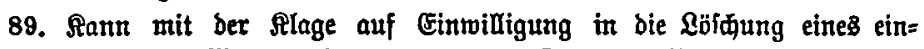

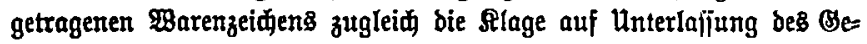

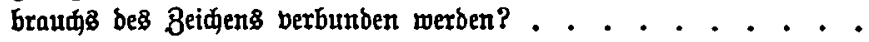

90. Breift gegenüber einer §lage, bie vor ber Erbteilung nur von einem Teile ber Diterben eine Berfügung über einen Madlag̈gegenftand be= gehnt, bie Cinrede mangelnder ßaffinlegitimation butra? Stellt bie

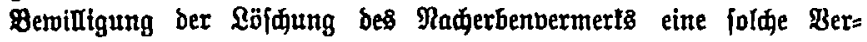
fügung bar? . . . . . . . . . . . . . . . . . . .

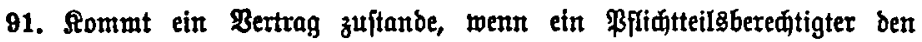

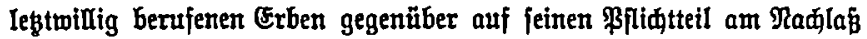

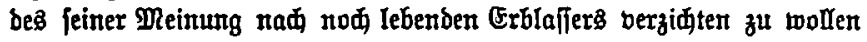

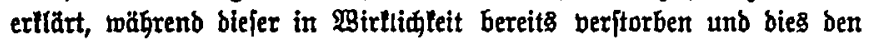
Erben belannt ift? . . . . . . . . . . . . . . . .

92. Bum Begrif ber Mahnung. Borausfezungen bes Berzug bei gegen= jeitigen Bertrăgen................... . 300

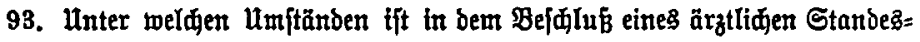
bereins, woburd feinen Mitgllebern ber beruflide Berlegr mit einem

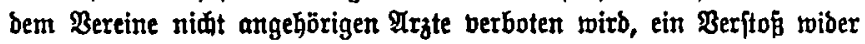

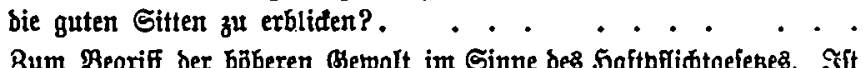

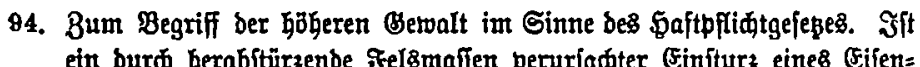

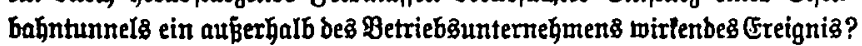


9it.

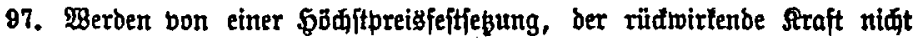

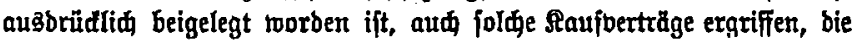

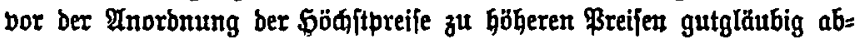

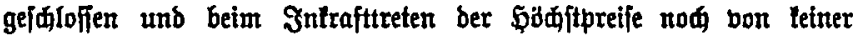
Seite erfüllt fino?

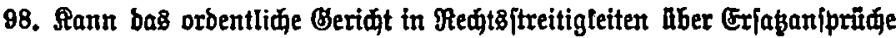

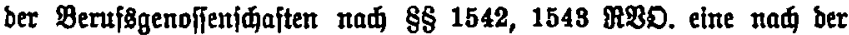

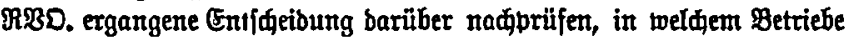
ber Berlebte tătig war? Benigen für bie भinmenbung bes \& 1543 Be=

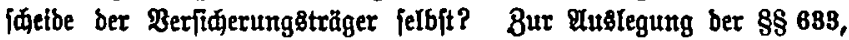
634 RBD. . . . . . . . . . . . . . . . . . . . .

100. Wie tann fid in Falle ber Rünoigung eines Gejelljafters beffen

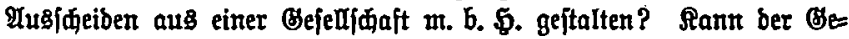
jellidafter gegenüber ber Einforberung ber Stammeinlage eintwenben, feine Sünbigung habe infolge Berfauldens ber Bejellfafaftsorgane niđjt

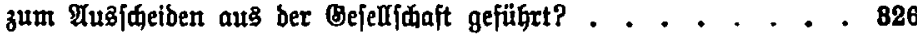

101. Bum Begrtffe der Befahr in Sinte bes \$477 BgB. . . . . . 880

102. Bedeutung ber Bufinerung, baß bie verfaufte \$are "bejめlagnafmes unb berwendungsfrei" jei . . . . . . . . . . . . . 882

103. \$añ bedürfen bie bei einem Erbaugeinanderfebungobertrage be

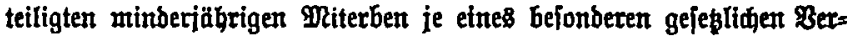

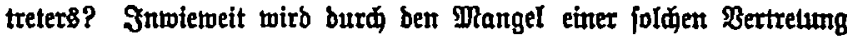
bie \$irtfamleit bes Bertrags beeinfunt? . . . . . . . . . 884

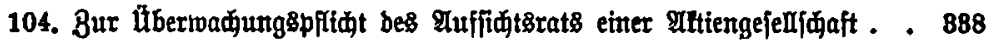

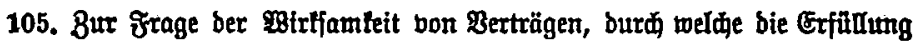

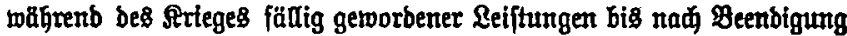
bes Strieges hinaugejejđoben toird . . . . . . . . . .

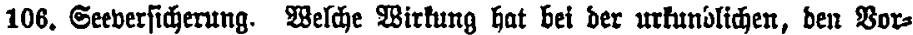

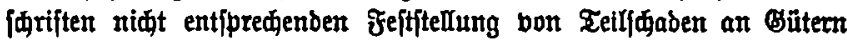

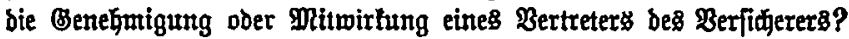

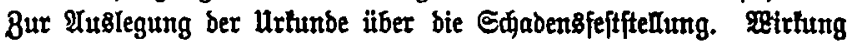
ber Jejtftellung . . . . . . . . . . . . . . . 344

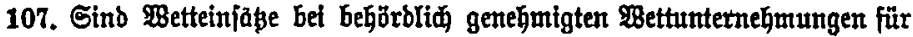

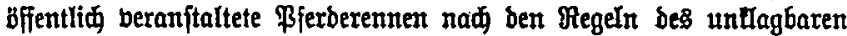
Spieles zu behandeln? . . . . . . . . . . . . . . . . 348

\section{b. Hantogedift.}

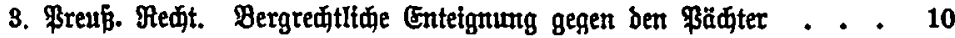

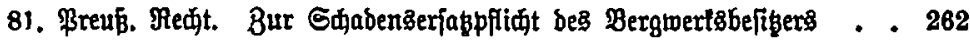

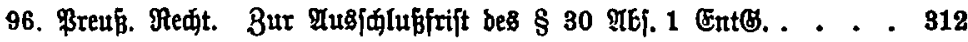




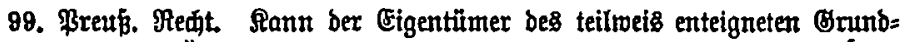

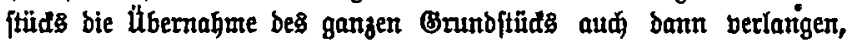

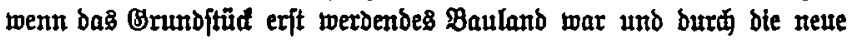

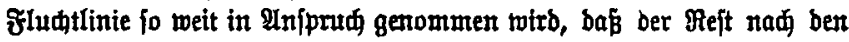

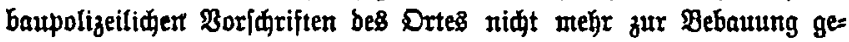
eigntet ift? . . . . . . . . . . . . . . .

\section{S̈ffentlide Redt.}

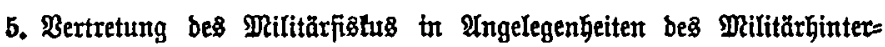
bliebenengeję̧es . . . . . . . . . . . . . . . . .

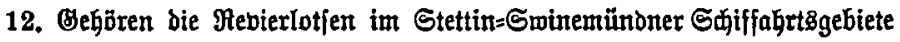

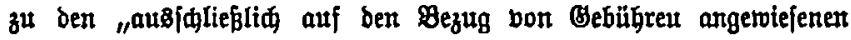
Beamten"? .

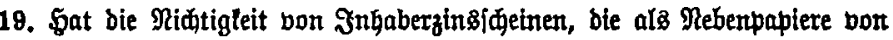

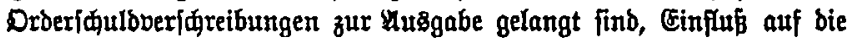
Būltigteit und Stempelpflidtigteit ber \$aubturtunden? \&ufredunung gegen ben Reidojtempel mit einer forbenung aud Beridulben eines Sterterbeamter.

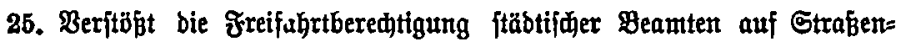
bahren gegen \& 21 preußj. Rleinbahn(8.?. . . . . . . . . .

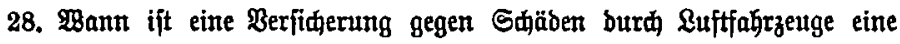
Zeutwerfiderang i. S. der Tarifur. 12 A RStemp8.? . . . . .

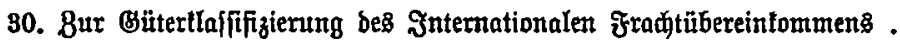

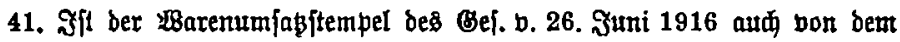
Betrage zut entriळten, ben ber \$erjtellex bon Bigaretten für bieje naథ 83 ßigSt(3. aufgemendet und bom Abnehmer exjtattet ergalten hat?

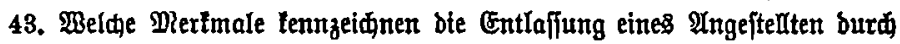

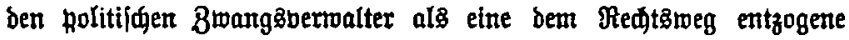
obrigfeitliče Anoronung? . . . . . . . . . . . . . 185

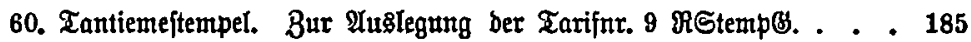

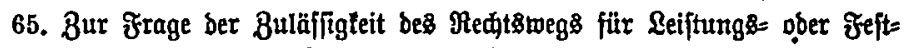
\{tellungstlagen, bie Strapenanliegerbetträge betreffen ...... 201

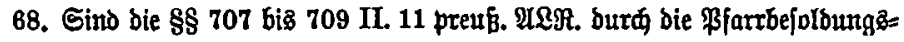
geję̧e v. 2. Julli 1898 unb 26. Mai 1909 abgeändert worben? . . 215

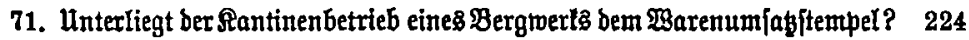

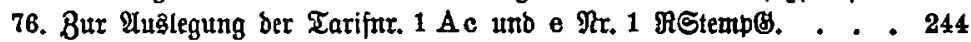

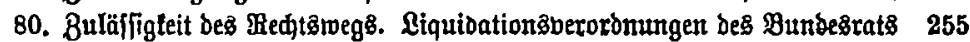

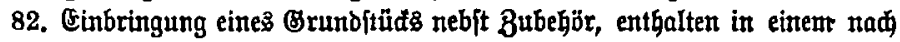
Dem 1. Oftober 1913 gejđloffenen Bettrag über Errifhtung einer offenten

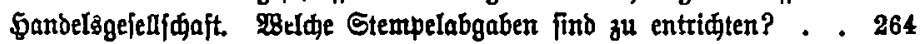

95. Berjorgung ber \$interbliebenen im ßriege Befallenter. . . . . 308 
SR.

\section{Bertidtides Berfabren.}

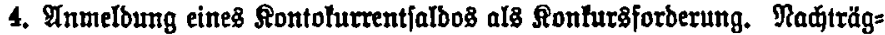

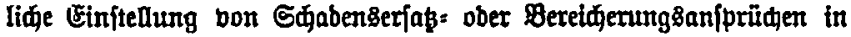
Das Montohurrent. . . . . . . . . . . . . . . . . 18

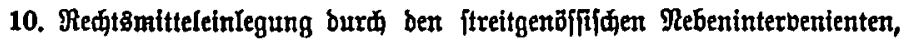
ber erfit nă 8uftellung bes urteils an bie \$artei beitritt . . . . 81

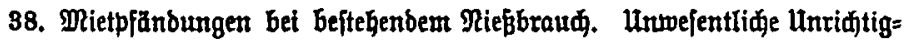

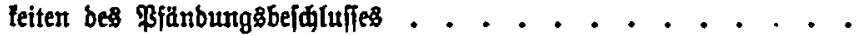

39. Ranr bas Berfahren bes Berulungsigeriđts bet Ermittelung ber tat= fädliden Borauşeß̧ungen einer DbFerbanz mit ber Mevifion bemängelt werben?. . . . . . . . . . . . . . . . . . .

40. Streit= unb Bejatwerdetwert. Bur Benteffung bes Streitwerts bei ber

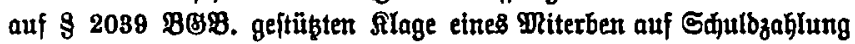

49. Unzuläfifige Beiđränlung ber Bethanblung auf ber Brunb beß silag=

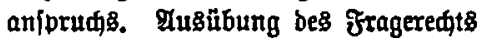

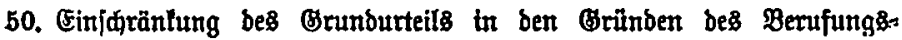
geriats; ift die झevifion juläfifg? . . . . . . . . . 156

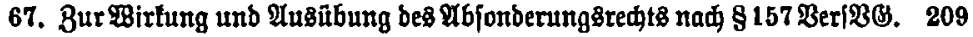

87. Rann ein Mitglieb bes Borftandes einer ßenfionstajje in einem Recht

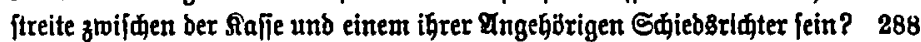

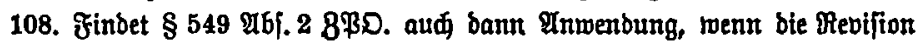

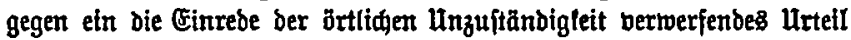

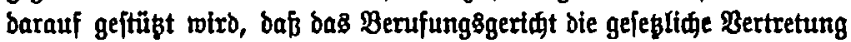

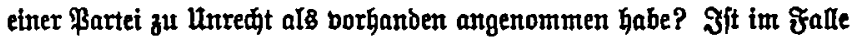

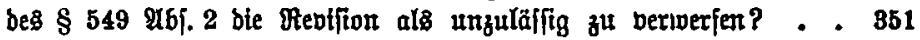

Sahregifter . . . . . . . . . . . . . . 953

Beję̧esregijter . . . . . . . . . . . . . . . 368

Bufammenftellung nad ber Beitfolge . . . . . . . . . 380

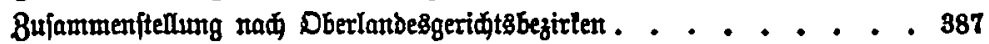

Betidtigungen. . . . . . . . . . . . . . . . . 888 